\title{
Selecting key toxins for focused development of elapid snake antivenoms and inhibitors guided by a Toxicity Score
}

\author{
Laustsen, Andreas Hougaard; Lohse, Brian; Lomonte, Bruno; Engmark, Mikael; Maria Gutierrez, Jose
}

Published in:

Toxicon

Link to article, DOI:

10.1016/j.toxicon.2015.07.334

Publication date:

2015

Document Version

Peer reviewed version

Link back to DTU Orbit

Citation (APA):

Laustsen, A. H., Lohse, B., Lomonte, B., Engmark, M., \& Maria Gutierrez, J. (2015). Selecting key toxins for focused development of elapid snake antivenoms and inhibitors guided by a Toxicity Score. Toxicon, 104, 4345. https://doi.org/10.1016/j.toxicon.2015.07.334

\section{General rights}

Copyright and moral rights for the publications made accessible in the public portal are retained by the authors and/or other copyright owners and it is a condition of accessing publications that users recognise and abide by the legal requirements associated with these rights.

- Users may download and print one copy of any publication from the public portal for the purpose of private study or research.

- You may not further distribute the material or use it for any profit-making activity or commercial gain

- You may freely distribute the URL identifying the publication in the public portal 
Selecting key toxins for focused development of elapid snake antivenoms and inhibitors guided by a Toxicity Score

${ }^{1}$ Department of Drug Design and Pharmacology, Faculty of Health and Medical Sciences, University of

*Corresponding author: José María Gutiérrez, Instituto Clodomiro Picado, Facultad de Microbiología, Universidad de Costa Rica, San José, Costa Rica; pone: 506-2511-7888; fax: 506-22920485; e mail: jose.gutierrez@ucr.ac.cr 
34 For more than 100 years, antivenoms have been produced by traditional methods of immunization of

35 large mammals with mixtures of snake venoms (WHO, 2010; Gutiérrez et al., 2011). With the introduction of proteomic and transcriptomic tools in the molecular analysis of both venoms (venomics) (Calvete, 2014) and antivenoms (antivenomics) (Calvete, 2011, Calvete et al., 2014), in combination with the toxicological assessment of venoms, a more in-depth understanding of venom composition and antivenom efficacy is being built. As retrieved from current public databases on Elapidae, values for Median Lethal Dose $\left(\mathrm{LD}_{50}\right)$ are known for 203 toxins, belonging to seven protein sub-families, originating from 40 species (Fig. 1). Furthermore, the number of elapids for which venom-wide proteomics or transcriptomics studies have been reported has now reached 49 out of 355 described species (our unpublished data; http://www.reptile-database.org). Information is now available for a considerable number of species of high medical relevance.

Taken together, these insights, and new information being published, may provide the grounds for a knowledge-based design of future antivenoms, based on the identification of the toxicologically most relevant toxins in venoms. Scientific efforts have so far mainly been centered around molecular targeting of relevant snake toxins by using synthetic inhibitors (de Oliveira et al., 2003, Howes et al., 2007), nanobodies (Richard et al., 2013), or antibody fragments (Kulkeaw et al., 2009, Tamarozzi et al., 2006). However, a limitation for introducing novel antitoxins or inhibitors is that they usually only target one or few individual toxins, and not the whole venom, which typically contains many toxins belonging to various protein families (Calvete, 2011). For many snake venoms, this challenge could be overcome if a complete overview of the effect of each toxin in the venom were at hand; this would allow a distinction between toxins that are essential to neutralize in envenoming cases, and toxins/proteins that are not pathophysiologically important. This could in the future enable researchers to develop antitoxins against the medically relevant toxins and use mixtures of these antitoxins as a replacement, or reinforcement, of antivenoms (Roncolato et al., 2013), potentially providing better efficacy and safety, and lower cost of treatment. We argue that despite the wealth of efforts within characterization of snake venoms, a lack of a systematic approach for evaluating the importance of individual toxins within whole venom still exists. Furthermore, no simple tool has been presented for evaluating whether a given toxin in a snake venom is of sufficient medical relevance to justify an antitoxin discovery program against it. Without such a tool, modern 
63 antivenom research based on molecular biology, medicinal chemistry, and biotechnology risks

64 becoming unfocused in the jungle of snake venom and antivenom data.

65 Primarily two properties of toxins are relevant when evaluating their potential medical

66 impact: 1) Intrinsic toxicity, and 2) abundance in venom. A systematic method for evaluating the relevance for acute toxicity, i.e. lethality, of each toxin in whole venom, taking both toxicity and abundance into account, was recently presented (Laustsen et al., 2015a). This method is based on a Toxicity Score, which is calculated for either a toxin or whole venom itself by dividing the relative toxin abundance in the venom (in percentage) by the $\mathrm{LD}_{50}$ value for the toxin in mice. If the confidence intervals are known for both the abundance and $\mathrm{LD}_{50}$, a confidence interval for the Toxicity Score can be easily calculated. This parameter was initially proposed for ranking the medical importance of toxins in the venom of the black mamba, Dendroaspis polylepis (Lautstsen et al., 2015a). Further studies on Naja kaouthia and Aipysurus laevis venoms strongly suggest that Toxicity Score is likely to be a better parameter to determine the medical relevance of a toxin than $L_{50}$ estimation alone, and may be used for assessing the relative importance of toxins in whole venom (Laustsen et al., 2015b, Laustsen et al., 2015c). The Toxicity Score can be used to quickly assess whether, for example, a toxin of high abundance and moderate toxicity is of higher importance than a very potent toxin of low abundance. This approach provides an easy, systematic method for identifying the key toxins that antivenom development should focus on. Table 1 presents an example of estimation of the Toxicity Score. an antivenom or an inhibitor, a decision has to be made to define a threshold value for relevance. For snakes that are able to inject large amounts of venom into their prey or predators, even toxins with a low Toxicity Score may become important. Therefore, the minimum cut-off value for the Toxicity

86 Score has to be analyzed on a case-by-case basis and has to take into account the total amount of venom that the specific snake is able to inject in a bite. Table 2 illustrates an example on how to determine the relevant toxins in a venom on the basis of a cut-off value for the Toxicity Score of 5. Using reported venom yields from milking of snakes (e.g. the values reported on http://snakedatabase.org/pages/LD50.php) provides a basis for the worst-case upper limit of venom

91 that may be injected in a bite from different snake species. For example, from our previous work on 
92 the venom of Naja kaouthia (Laustsen et al., 2015b), reported in this database to deliver up to $742 \mathrm{mg}$

93 of venom, a cut-off value of the Toxicity Score of 5 would select a group of 5 neurotoxins of the three-

94 finger toxin family and one $\mathrm{PLA}_{2}$ among a total of 28 fractions as the most relevant targets to

95 neutralize. Fractions with a Toxicity Score below this value would have less than $37 \mathrm{LD}_{50} \mathrm{~S}$ for an

96 envenoming with the maximum yield of $742 \mathrm{mg}$, and are thus likely not to play a significant role in

97 overall venom toxicity.

98 The issue of potential synergism between venom components has to be taken into 99 consideration when selecting key toxins to focus antivenom development efforts on. Using the 100 Toxicity Score allows for the identification of venoms where toxins display synergistic effects. As 101 shown in the cases of D. polylepis (Laustsen et al., 2015a) and A. laevis (Laustsen et al., 2015c)

102 venoms, the Toxicity Score of the whole venom is higher than the sum of Toxicity Scores of individual 103 toxins, thus indicating the presence of synergism. In these cases it is relevant to further investigate 104 the synergistic effects by assessing the toxicities of pairs of toxins in order to identify proteins of low 105 Toxicity Score, but which exert synergistic effects. An example of such an approach is given by 106 Strydom (1976) for toxins from D. polylepis venom. Despite their low intrinsic toxicity, synergistically 107 acting toxins would also be good candidates to include in an antivenom development program.

108 The Toxicity Score can be readily applied to the study of elapid snake venoms because: (a)

109 the main toxic activity of these venoms is neuromuscular paralysis leading to respiratory arrest and

110 death, thus making the assessment of lethality a clinically-relevant parameter; (b) the solvents used in

111 many separation methods (such as the commonly used reverse-phase HPLC) do not generally 112 denature the most relevant elapid venom toxins, such as three-finger toxins, dendrotoxins, and 113 phospholipases $\mathrm{A}_{2}\left(\mathrm{PLA}_{2}\right.$ ) (Fry et al., 2015); and (c) the mouse model used in the determination of $\mathrm{LD}_{50}$

114 is generally relevant to the human situation, as the physiological mechanisms involved in 115 neuromuscular transmission in mice and humans are similar.

116 The use of this parameter in viperid snake venoms might be more complicated, for the 117 following reasons: (a) Although lethality is the most serious complication of viperid envenomings, 118 other aspects also have high medical relevance, such as local tissue damage, i.e. necrosis and 119 hemorrhage, and systemic effects such as coagulopathy and systemic hemorrhage (Warrell, 2010). (b) 120 The solvents used in reverse phase-HPLC, especially acetonitrile, denature relevant toxins in viperid 
121 venoms, particularly zinc-dependent metalloproteinases (SVMPs), thus affecting their toxicity. It is

122 likely, nevertheless, that these two hurdles might be overcome in the future, with the development of 123 non-denaturing separation methods of high resolution. At present, using the Toxicity Score is

124 therefore only feasible for focusing development efforts on elapid antivenoms, where the toxins do not loose activity in the purification process. An exception is the case of some Australian elapid

126 venoms containing potent procoagulant serine proteinases playing a significant role in toxicity (Kini, 127 2005), which could be affected by chromatographic solvents; however, the key toxins in the vast 128 majority of elapid venoms can withstand solvents used in reverse-phase HPLC.

129 The Toxicity Score has been employed for directing drug discovery efforts against $\alpha$ 130 cobratoxin, which was shown to have the highest relevance in Naja kaouthia venom (Laustsen et al., 131 2015b), using phage display screening (Laustsen et al., 2015d). In this example, the Toxicity Score predicts that blocking the action of $\alpha$-cobratoxin will abrogate the overall toxicity of the venom. In

133 conclusion, when working with elapid venoms, using the Toxicity Score may thus provide a clearer 134 path for determining the medical importance of different toxins in whole venom for the development 135 of toxin inhibitors and the improvement of antivenoms.

References

138 Calvete, J.J., 2014. Next-generation snake venomics: Protein-locus resolution through venom 139 proteome decomplexation. Expert Rev. Proteomics 11, 315-329.

140 Calvete, J.J., 2011. Proteomic tools against the neglected pathology of snake bite envenoming. Expert 141 Rev. Proteomics 8, 739-758.

142 Calvete, J.J., Sanz, L., Pla, D., Lomonte, B., Gutiérrez, J.M., 2014. Omics meets biology: application to the design and preclinical assessment of antivenoms. Toxins 6, 3388-3405.

144 de Oliveira, M., Cavalcante, W.L., Arruda, E.Z., Melo, P.A., Silva, M.D.P., Galacci, M., 2003. Antagonism of myotoxic and paralyzing activities of bothropstoxin-I by suramin. Toxicon 42, 373-379.

146 Fry, B.G., Undheim, E.A.B., Jackson, T.N.W., Roelants, K., Georgieva, D., Vetter, I., Calvete, J.J., Scheib, 147 H., Cribb, B.W., Yang, D.C., Daly, N.L., Manchadi, M.L.R., Gutiérrez, J.M., Lomonte, B., Nicholson, 148 G.M., Dziemborowicz, S., Lavergne, V., Ragnarsson, L., Rash, L.D., Mobli, M., Hodgson, W.C., 

Oxford, pp. 153-214.

Gutiérrez, J.M., León, G., Lomonte, B., Angulo, Y., 2011. Antivenoms for snakebite envenomings.

Howes, J.M., Theakston, R.D.G., Laing, G.D., 2007. Neutralization of the haemorrhagic activities of viperine snake venoms and venom metalloproteinases using synthetic peptide inhibitors and chelators. Toxicon 49, 734-739.

Kini, R.M., 2005. The intriguing world of prothrombin activators from snake venom. Toxicon 45, 11331145.

Kulkeaw, K., Sakolvaree, Y., Srimanote, P., Tongtawe, P., Maneewatch, S., Sookrung, N., Tungtrongchitr, A., Tapchaisri, P., Kurazono, H., Chaicumpa, W., 2009. Human monoclonal ScFv neutralize lethal Thai cobra, Naja kaouthia, neurotoxin. J. Proteomics 72, 270-282.

Laustsen, A.H., Lomonte, B., Lohse, B., Fernández, J., Gutiérrez, J.M., 2015a. Unveiling the nature of black mamba (Dendroaspis polylepis) venom through venomics and antivenom immunoprofiling: Identification of key toxin targets for antivenom development. J. Proteomics 119, 126-142. 2015b. Snake venomics of monocled cobra (Naja kaouthia) and investigation of human IgG response against venom toxins. Toxicon 99, 23-35.

Laustsen, A.H, Gutiérrez, J.M., Rasmussen, A.R., Engmark, M., Gravlund, P., Saunders, K.L., Lohse, B.,

172 Laustsen, A.H., Lynagh, T., Kringelum, J., Christensen, A., Johannesen, J., Engmark, M., Pless, S.A., 173 Olsen, L., Fernández, J., Gutiérrez, J.M., Lomonte, B., Lohse, B. (2015-05-21). Discovery of 174 Peptidic Anti-cobratoxins by Next Generation Phage Display. PhD Day 2015, Faculty of Health Sciences, Copenhagen University. 
176 Richard, G., Meyers, A.J., McLean, M.D., Arbabi-Ghahroudi, M., MacKenzie, R., Hall, J.C., 2013. In vivo 177 neutralization of alpha-cobratoxin with high-affinity llama single-domain antibodies $\left(\mathrm{V}_{\mathrm{H}} \mathrm{Hs}\right)$ and a $178 \quad V_{\mathrm{H}} \mathrm{H}-\mathrm{Fc}$ antibody. PLoS One 8, e69495.

179 Roncolato, E.C., Pucca, M.B., Funayama, J.C., Bertolini, T.B., Campos, L.B., Barbosa, J.E., 2013. Human 180 antibody fragments specific for Bothrops jararacussu venom reduce the toxicity of other 181 Bothrops sp venoms. J. Immunotoxicol 10, 160-168.

182 Strydom, D.J., 1976. Snake venom toxins - purification and properties of low-molecular-weight 183 polypeptides of Dendroaspis polylepis (black mamba) venom. Eur. J. Biochem. 69, 169-76.

184 Tamarozzi, M.B., Soares, S.G., Marcussi, S., Giglio, J.R., Barbosa, J.E., 2006. Expression of recombinant 185 human antibody fragments capable of inhibiting the phospholipase and myotoxic activities of 186 Bothrops jararacussu venom. Biochim. Biophys. Acta 1760, 1450-1457.

187 Warrell, D.A., 2010. Snake bite. Lancet 375(9708), 77-88.

188 World Health Organization, 2010. WHO Guidelines for the Production, Control and Regulation of 189 Snake Antivenom Immunoglobulins. Geneva: WHO, 134 p.

190

191 
Table 1: Example of how Toxicity Scores are calculated for three toxins and whole venom.

193

\begin{tabular}{|l|c|c|c|}
\hline & Abundance (\%) & $\left.\mathbf{L D}_{\mathbf{5 0}} \mathbf{( m g} / \mathbf{k g}\right)$ & Toxicity Score* \\
\hline Toxin 1 & 10 & 0.1 & 100 \\
\hline Toxin 2 & 20 & 2 & 10 \\
\hline Toxin 3 & 50 & 1 & 50 \\
\hline Whole venom & 100 & 0.25 & 400 \\
\hline
\end{tabular}

194

195

${ }^{*}$ Toxicity Score $=\frac{\text { Abundance }(\%)}{L D_{50}}$

196

197

198

199

200

201

202

203

204

205

206

207

208

209

210

211

212

213

214 
215 Table 2: Example of how Toxicity Scores may help distinguish between medically relevant and non-

216 relevant toxins/proteins, when selecting targets for focused antivenom development.

\begin{tabular}{|l|c|c|c|c|}
\hline & Abundance (\%) & LD $_{50}(\mathrm{mg} / \mathrm{kg})$ & Toxicity Score & Medically relevant \\
\hline Whole venom & 100 & 0.25 & 400 & \\
\hline Toxin A & 10 & 0.05 & 200 & Yes \\
\hline Toxin B & 20 & 0.2 & 100 & Yes \\
\hline Toxin C & 50 & 1 & 50 & No \\
\hline Toxin D & 10 & 10 & 1 & No \\
\hline Toxin E & 10 & 10 & 1 & Y. \\
\hline
\end{tabular}


235 Figure legend

236 Figure 1: Overview of numbers of elapid snake venom toxins for which toxicity, as judged by

237 estimation of Median Lethal Dose $\left(\mathrm{LD}_{50}\right)$, has been assessed. Snake genera are highlighted in $\mathbf{A}$ by 238 color codes.

239

240

241 

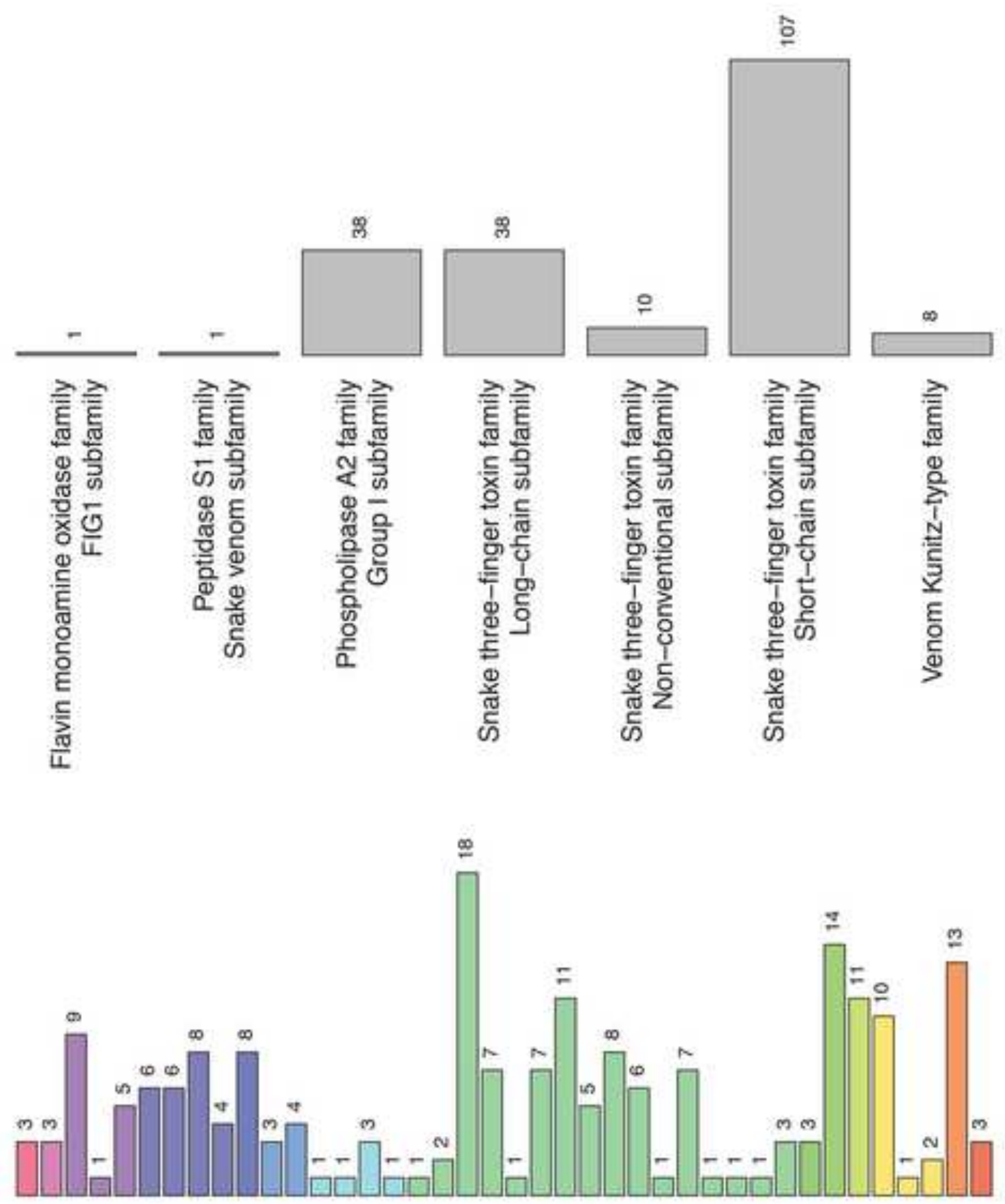

ڤผ

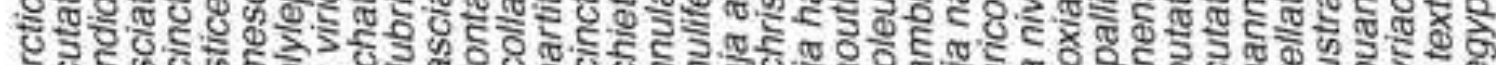
过 कू

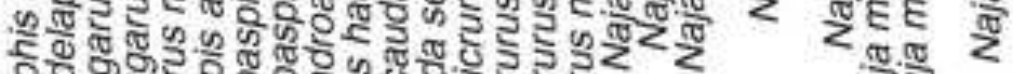
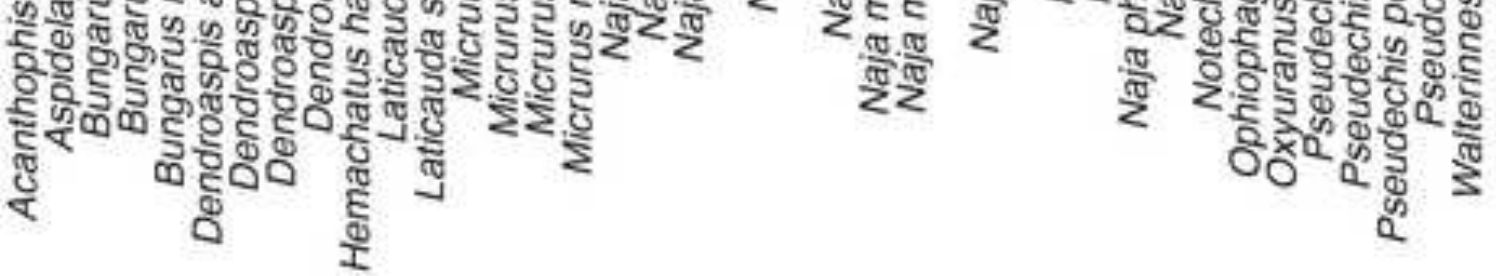